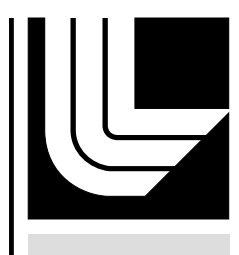

LAW REN CE LIVERMORE N A T IO N A L LABORATORY

Wave-Based Inversion \& Imaging for the Optical Quadrature Microscope

S. K. Lehman

November 14, 2005 
This document was prepared as an account of work sponsored by an agency of the United States Government. Neither the United States Government nor the University of California nor any of their employees, makes any warranty, express or implied, or assumes any legal liability or responsibility for the accuracy, completeness, or usefulness of any information, apparatus, product, or process disclosed, or represents that its use would not infringe privately owned rights. Reference herein to any specific commercial product, process, or service by trade name, trademark, manufacturer, or otherwise, does not necessarily constitute or imply its endorsement, recommendation, or favoring by the United States Government or the University of California. The views and opinions of authors expressed herein do not necessarily state or reflect those of the United States Government or the University of California, and shall not be used for advertising or product endorsement purposes.

This work was performed under the auspices of the U.S. Department of Energy by University of California, Lawrence Livermore National Laboratory under Contract W-7405-Eng-48. 


\title{
Wave-Based Inversion \& Imaging for the Optical Quadrature Microscope
}

\author{
Sean K. Lehman
}

November 14, 2005

\begin{abstract}
The Center for Subsurface Sensing \& Imaging System's (CenSSIS) Optical Quadrature Microscope (OQM) is a narrow band visible light microscope capable of measuring both amplitude and phase of a scattered field. We develop a diffraction tomography, that is, wavebased, scattered field inversion and imaging algorithm, for reconstructing the refractive index of the scattering object.
\end{abstract}




\section{Contents}

1 Introduction $\quad 1$

2 Total Field Propagation $\quad 1$

2.1 Born Approximation . . . . . . . . . . . . . . . . . . . 3

2.2 Rytov Approximation . . . . . . . . . . . . . . . . . . 4

2.3 Summary of Born \& Rytov Approximations . . . . . . . . . . . . . . . 6

2.4 Incident Fields . . . . . . . . . . . . . . . . . . . . 6

3 Inversion Using Incident Plane Wave and Planar Measurement 7

3.1 Plane Wave Details \& Fourier Diffraction Theorem . . . . . . . . . . . . . . . 7

3.2 Plane Wave Inversion . . . . . . . . . . . . . . . . . . . . 12

4 Summary \& Conclusions $\quad 14$

A Standard Fourier Transforms $\quad 16$ 


\section{Introduction}

The Center for Subsurface Sensing \& Imaging System's (CenSSIS) Optical Quadrature Microscope (OQM) is a narrow band visible light microscope capable of measuring both amplitude and phase of a scattered field. We develop a diffraction tomography, that is, wave-based, scattered field inversion and imaging algorithm, for reconstructing the refractive index of the scattering object.

The purposes of this report is to develop a wave-based inversion and imaging algorithm, not to describe the OQM. We refer the reader to the references $[1,2,3,4,5]$ for a thorough development of the OQM. Our fundamental assumption, here, is that the OQM can measure a fully complex field (simultaneously measure the magnitude and phase of a scattered field) at a single measurement plane. We also assume the incident field is planar and that both it and measurement plane are rotated about the scattering object (object under evaluation) at multiple angular locations uniformly distributed over $360^{\circ}$ as indicated in Figure 1.

We develop our forward scattering model in the next section including derivations under the Born (amplitude) and Rytov (phase) approximations. The inversion algorithm is described in Section 3.

\section{Total Field Propagation}

We begin by considering the wave equation

$$
\left[\nabla^{2}-\frac{n^{2}(\mathbf{r})}{c_{0}^{2}} \partial_{t}^{2}\right] \psi^{t o t}\left(\mathbf{r}, \mathbf{R}_{n}^{t}, t\right)=-p\left(\mathbf{r}, \mathbf{R}_{n}^{t}, t\right),
$$

in which we have assumed a variable medium through the refractive index

$$
n(\mathbf{r}) \equiv \frac{c_{0}}{c(\mathbf{r})}
$$

$n(\mathbf{r})$ to represents the presence of a scattering object, that is, the object under evaluation. $\psi^{\text {tot }}\left(\mathbf{r}, \mathbf{R}_{n}^{t}, t\right)$ is the total field (the sum of the scattered and incident fields), $p\left(\mathbf{r}, \mathbf{R}_{n}^{t}, t\right)$ is the incident pulse applied to the $n$-th transmitter located at $\mathbf{R}_{n}^{t}$, and $c_{0}$ is the background wave speed. We Fourier transform Eqn. 1 using the temporal transform of Appendix A:

$$
\left[\nabla^{2}+k_{0}^{2} n^{2}(\mathbf{r})\right] \psi^{t o t}\left(\mathbf{r}, \mathbf{R}_{n}^{t}, \omega\right)=-p\left(\mathbf{r}, \mathbf{R}_{n}^{t}, \omega\right)
$$

where the background wavenumber is defined as

$$
k_{0} \equiv \frac{\omega}{c_{0}}
$$

When solving the forward problem, it is frequently convenient to cast Eqn. 3 into an integral equation. We do so by adding $k_{0} \psi^{\text {tot }}\left(\mathbf{r}, \mathbf{R}_{n}^{t}, \omega\right)$ to both sides of Eqn. 3, and moving the inhomogeneous term to the right hand side:

$$
\left[\nabla^{2}+k_{0}^{2}\right] \psi^{t o t}\left(\mathbf{r}, \mathbf{R}_{n}^{t}, \omega\right)=-p(\mathbf{r})-\left[k_{0}^{2} n^{2}(\mathbf{r})-k_{0}^{2}\right] \psi^{t o t}\left(\mathbf{r}, \mathbf{R}_{n}^{t}, \omega\right) .
$$


Define the object function as

$$
o(\mathbf{r}) \equiv n^{2}(\mathbf{r})-1
$$

and express Eqn. 4 as

$$
\left[\nabla^{2}+k_{0}^{2}\right] \psi^{t o t}\left(\mathbf{r}, \mathbf{R}_{n}^{t}, \omega\right)=-p\left(\mathbf{r}, \mathbf{R}_{n}^{t}, \omega\right)-k_{0}^{2} o(\mathbf{r}) \psi^{t o t}\left(\mathbf{r}, \mathbf{R}_{n}^{t}, \omega\right)
$$

The first term on the right-hand side of Eqn. $6, p\left(\mathbf{r}, \mathbf{R}_{n}^{t}, \omega\right)$, is the primary source. The second, source-like, term on the right-hand side, $k_{0}^{2} o(\mathbf{r}) \psi^{\text {tot }}\left(\mathbf{r}, \mathbf{R}_{n}^{t}, \omega\right)$, is known as the secondary source. We may use Green's theorem to cast the differential equation of Eqn. 6 into an integral equation [6],

$$
\begin{aligned}
\psi^{\text {tot }}\left(\mathbf{r}, \mathbf{R}_{n}^{t}, \omega\right)= & \underbrace{\int d \mathbf{r}^{\prime} G_{0}\left(\mathbf{r}, \mathbf{r}^{\prime}, \omega\right) p\left(\mathbf{r}^{\prime}, \mathbf{R}_{n}^{t}, \omega\right)}_{\psi^{\text {inc }}\left(\mathbf{r}, \mathbf{R}_{n}^{t}, \omega\right)}+ \\
& \underbrace{k_{0}^{2} \int d \mathbf{r}^{\prime} G_{0}\left(\mathbf{r}, \mathbf{r}^{\prime}, \omega\right) o\left(\mathbf{r}^{\prime}\right) \psi^{t o t}\left(\mathbf{r}^{\prime}, \mathbf{R}_{n}^{t}, \omega\right)}_{\psi^{\text {scat }}\left(\mathbf{r}, \mathbf{R}_{n}^{t}, \omega\right)},
\end{aligned}
$$

where the background Green function satisfies

$$
\left[\nabla^{2}+k_{0}^{2}\right] G_{0}\left(\mathbf{r}, \mathbf{r}^{\prime}, \omega\right)=-\delta\left(\mathbf{r}-\mathbf{r}^{\prime}\right)
$$

We define the primary, incident, or background field as

$$
\psi^{i n c}\left(\mathbf{r}, \mathbf{R}_{n}^{t}, \omega\right) \equiv \int d \mathbf{r}^{\prime} G_{0}\left(\mathbf{r}, \mathbf{r}^{\prime}, \omega\right) p\left(\mathbf{r}^{\prime}, \mathbf{R}_{n}^{t}, \omega\right)
$$

so that Eqn. 7 reads

$$
\psi^{t o t}\left(\mathbf{r}, \mathbf{R}_{n}^{t}, \omega\right)=\psi^{i n c}\left(\mathbf{r}, \mathbf{R}_{n}^{t}, \omega\right)+k_{0}^{2} \int d \mathbf{r}^{\prime} G_{0}\left(\mathbf{r}, \mathbf{r}^{\prime}, \omega\right) o\left(\mathbf{r}^{\prime}\right) \psi^{t o t}\left(\mathbf{r}^{\prime}, \mathbf{R}_{n}^{t}, \omega\right)
$$

The scattered field is then defined as the difference between the total and incident fields,

$$
\begin{aligned}
\psi^{\text {scat }}\left(\mathbf{r}, \mathbf{R}_{n}^{t}, \omega\right) & \equiv \psi^{t o t}\left(\mathbf{r}, \mathbf{R}_{n}^{t}, \omega\right)-\psi^{i n c}\left(\mathbf{r}, \mathbf{R}_{n}^{t}, \omega\right) \\
& =k_{0}^{2} \int d \mathbf{r}^{\prime} G_{0}\left(\mathbf{r}, \mathbf{r}^{\prime}, \omega\right) o\left(\mathbf{r}^{\prime}\right) \psi^{t o t}\left(\mathbf{r}^{\prime}, \mathbf{R}_{n}^{t}, \omega\right)
\end{aligned}
$$

We observe that with the primary field satisfying

$$
\left[\nabla^{2}+k_{0}^{2}\right] \psi^{i n c}\left(\mathbf{r}, \mathbf{R}_{n}^{t}, \omega\right)=-p\left(\mathbf{r}, \mathbf{R}_{n}^{t}, \omega\right)
$$

the scattered field obeys

$$
\left[\nabla^{2}+k_{0}^{2}\right] \psi^{\text {scat }}\left(\mathbf{r}, \mathbf{R}_{n}^{t}, \omega\right)=-k_{0}^{2} o(\mathbf{r}) \psi^{t o t}\left(\mathbf{r}, \mathbf{R}_{n}^{t}, \omega\right),
$$


or alternatively,

$$
\left[\nabla^{2}+k_{0}^{2} n^{2}(\mathbf{r})\right] \psi^{s c a t}\left(\mathbf{r}, \mathbf{R}_{n}^{t}, \omega\right)=-k_{0}^{2} o(\mathbf{r}) \psi^{i n c}\left(\mathbf{r}, \mathbf{R}_{n}^{t}, \omega\right) .
$$

The integral equation of Eqn. 11 is non-linear in that the scattered field appears in both sides of the equation. The differential equation of Eqn. 14 offers the problem of having spatially dependent scattering operator. Exact analytic solutions for the scattered field exist only for a limited set of scattering objects. Numerical solutions are readily available but not appropriate for an arbitrary object tomographic inversion algorithm. Thus, we wish to simplify our forward model. In the following section we simplify the model under the Born approximation. In Section 2.2 use the Rytov approximation. We note that the Born and Rytov approximations are the two most practical simplifications when little is known of the scattering object. When more is known of the object, this prior information can be included in the model via a modified Green function or through a model-based processing approach and an (fast) forward propagator.

\subsection{Born Approximation}

One potential method of simplifying Eqn. 14 for solving is to use a perturbation approach. We express the refractive index and scattered field as [7]

$$
\begin{aligned}
n^{2}(\mathbf{r}) & =n_{0}^{2}(\mathbf{r})+\underbrace{\epsilon n_{1}(\mathbf{r})+\epsilon^{2} n_{2}(\mathbf{r})+\cdots}_{\equiv \delta n(\mathbf{r})}, \\
\psi^{s c a t}\left(\mathbf{r}, \mathbf{R}_{n}^{t}, \omega\right) & =\psi_{D W B}^{s c a t}\left(\mathbf{r}, \mathbf{R}_{n}^{t}, \omega\right)+\epsilon \psi_{1}^{s c a t}\left(\mathbf{r}, \mathbf{R}_{n}^{t}, \omega\right)+\epsilon^{2} \psi_{2}^{s c a t}\left(\mathbf{r}, \mathbf{R}_{n}^{t}, \omega\right)+\cdots,
\end{aligned}
$$

respectively, substitute them into Eqn. 14, and equate like powers of $\epsilon$. Doing so we obtain the follow set of equations:

$$
\begin{aligned}
{\left[\nabla^{2}+k_{0}^{2} n_{0}^{2}(\mathbf{r})\right] \psi_{D W B}^{\text {scat }}\left(\mathbf{r}, \mathbf{R}_{n}^{t}, \omega\right)=} & -k_{0}^{2} o(\mathbf{r}) \psi^{\text {inc }}\left(\mathbf{r}, \mathbf{R}_{n}^{t}, \omega\right), \\
{\left[\nabla^{2}+k_{0}^{2} n_{0}^{2}(\mathbf{r})\right] \psi_{1}^{\text {scat }}\left(\mathbf{r}, \mathbf{R}_{n}^{t}, \omega\right)=} & -k_{0}^{2} n_{1}(\mathbf{r}) \psi_{D W B}^{s c a t}\left(\mathbf{r}, \mathbf{R}_{n}^{t}, \omega\right), \\
{\left[\nabla^{2}+k_{0}^{2} n_{0}^{2}(\mathbf{r})\right] \psi_{2}^{\text {scat }}\left(\mathbf{r}, \mathbf{R}_{n}^{t}, \omega\right)=} & -k_{0}^{2} n_{2}(\mathbf{r}) \psi_{D W B}^{\text {scat }}\left(\mathbf{r}, \mathbf{R}_{n}^{t}, \omega\right) \\
& -k_{0}^{2} n_{1}(\mathbf{r}) \psi_{1}^{\text {scat }}\left(\mathbf{r}, \mathbf{R}_{n}^{t}, \omega\right), \\
\vdots & \\
{\left[\nabla^{2}+k_{0}^{2} n_{0}^{2}(\mathbf{r})\right] \psi_{l}^{\text {scat }}\left(\mathbf{r}, \mathbf{R}_{n}^{t}, \omega\right)=} & -k_{0}^{2} \sum_{m=0}^{l-1} n_{l-m}(\mathbf{r}) \psi_{m}^{\text {scat }}\left(\mathbf{r}, \mathbf{R}_{n}^{t}, \omega\right) .
\end{aligned}
$$

The Green function for the left hand side operators of Eqns. 16 through 18 satisfies

$$
\left[\nabla^{2}+k_{0}^{2} n_{0}^{2}(\mathbf{r})\right] G\left(\mathbf{r}, \mathbf{r}^{\prime}, \omega\right)=-\delta\left(\mathbf{r}-\mathbf{r}^{\prime}\right) .
$$

Note: this is not the background Green function of Eqn. 8. Using this to cast Eqn. 16 into an integral equation, we obtain the distorted wave Born approximation to the scattered field,

$$
\psi_{D W B}^{\text {scat }}\left(\mathbf{r}, \mathbf{R}_{n}^{t}, \omega\right)=k_{0}^{2} \int d \mathbf{r}^{\prime} G\left(\mathbf{r}, \mathbf{r}^{\prime}, \omega\right) o\left(\mathbf{r}^{\prime}\right) \psi^{i n c}\left(\mathbf{r}^{\prime}, \mathbf{R}_{n}^{t}, \omega\right) .
$$


When $n_{0}(\mathbf{r}) \equiv 1$, this reduces to the standard Born approximation [8],

$$
\psi_{B}^{s c a t}\left(\mathbf{r}, \mathbf{R}_{n}^{t}, \omega\right)=k_{0}^{2} \int d \mathbf{r}^{\prime} G_{0}\left(\mathbf{r}, \mathbf{r}^{\prime}, \omega\right) o\left(\mathbf{r}^{\prime}\right) \psi^{i n c}\left(\mathbf{r}^{\prime}, \mathbf{R}_{n}^{t}, \omega\right)
$$

Comparing this to Eqn. 11, we see the Born approximation effectively replaces the total field with the incident field.

\subsection{Rytov Approximation}

The Rytov approximation [8] starts with Eqn. 6 and the assumptions that the source term is either identically zero (accurate if we consider a volume which excludes the source support),

$$
p\left(\mathbf{r}, \mathbf{R}_{n}^{t}, \omega\right) \equiv 0
$$

or a plane wave with propagation vector $\hat{\mathbf{s}}$,

$$
p(\mathbf{r}, \hat{\mathbf{s}}, \omega) \equiv P(\omega) e^{i k_{0} \hat{\mathbf{s}} \cdot \mathbf{r}}
$$

and that the total field can be expressed as

$$
\psi^{\text {tot }}(\mathbf{r})=\psi^{i n c}(\mathbf{r}) \psi^{\text {scat }}(\mathbf{r})=e^{\phi_{i}(\mathbf{r})} e^{\phi_{s}(\mathbf{r})}
$$

where $\phi_{i}(\mathbf{r})$ and $\phi_{s}(\mathbf{r})$ are the incident and scattered field complex phases, respectively, and the total phase is

$$
\phi(\mathbf{r})=\phi_{i}(\mathbf{r})+\phi_{s}(\mathbf{r})
$$

For this section, we omit the explicit dependence of the fields on the temporal frequency, $\omega$, and references to the incident field ( $\mathbf{R}_{n}^{t}$ for a point source, or $\hat{\mathbf{s}}$ for a plane wave). We have the following identities

$$
\begin{aligned}
\nabla \psi^{t o t}(\mathbf{r}) & =\psi^{t o t}(\mathbf{r}) \nabla \phi(\mathbf{r}) \\
\nabla^{2} \psi^{t o t}(\mathbf{r}) & =\psi^{t o t}(\mathbf{r})\left[\nabla^{2} \phi(\mathbf{r})+(\nabla \phi(\mathbf{r}))^{2}\right]
\end{aligned}
$$

Using Eqn. 28, Eqn. 6 reads,

$$
\nabla^{2} \phi(\mathbf{r})+(\nabla \phi(\mathbf{r}))^{2}+k_{0}^{2}=-k_{0}^{2} o(\mathbf{r})
$$

which reduces to

$$
\nabla^{2} \phi(\mathbf{r})+(\nabla \phi(\mathbf{r}))^{2}=-k_{0}^{2} n^{2}(\mathbf{r})
$$

where we have used the definition of the object function from Eqn. 5. 
As in the Born approximation development, we expand the object function and total phase as follows,

$$
\begin{aligned}
n^{2}(\mathbf{r}) & =n_{0}^{2}(\mathbf{r})+\underbrace{\epsilon n_{1}(\mathbf{r})+\epsilon^{2} n_{2}(\mathbf{r})+\cdots}_{\equiv \delta n(\mathbf{r})} \\
\phi(\mathbf{r}) & =\phi_{i}(\mathbf{r})+\epsilon \phi_{1}^{s c a t}(\mathbf{r})+\epsilon^{2} \phi_{2}^{s c a t}(\mathbf{r})+\cdots .
\end{aligned}
$$

Substituting the expansions from Eqn. 31 into Eqn. 30 and collecting powers of $\epsilon$, we find

$$
\begin{aligned}
\nabla^{2} \phi_{i}+\left(\nabla \phi_{i}\right)^{2} & =-k_{0}^{2} n_{0}^{2}(\mathbf{r}), \\
\nabla^{2} \phi_{1}+2 \nabla \phi_{i} \cdot \nabla \phi_{1} & =-k_{0}^{2} n_{1}(\mathbf{r}), \\
\nabla^{2} \phi_{2}+2 \nabla \phi_{i} \cdot \nabla \phi_{2} & =-k_{0}^{2} n_{2}(\mathbf{r})-\left(\nabla \phi_{1}\right)^{2}, \\
\nabla^{2} \phi_{3}+2 \nabla \phi_{i} \cdot \nabla \phi_{3} & =-k_{0}^{2} n_{3}(\mathbf{r})-2 \nabla \phi_{1} \cdot \nabla \phi_{2}, \\
& \vdots \\
\nabla^{2} \phi_{l}+2 \nabla \phi_{i} \cdot \nabla \phi_{l} & =-k_{0}^{2} n_{l}(\mathbf{r})-\sum_{m=1}^{l-1} \nabla \phi_{m} \cdot \nabla \phi_{l-m} .
\end{aligned}
$$

Consider

$$
\begin{aligned}
\nabla^{2}\left(\psi^{i n c}(\mathbf{r}) \phi_{s}(\mathbf{r})\right)= & \left(\psi^{i n c}(\mathbf{r}) \phi_{s}(\mathbf{r})\right)\left[\left(\nabla \phi_{i}(\mathbf{r})\right)^{2}+\nabla^{2} \phi_{i}(\mathbf{r})\right] \\
& +\psi^{i n c}(\mathbf{r})\left[2 \nabla \phi_{i}(\mathbf{r}) \cdot \nabla \phi_{s}(\mathbf{r})+\nabla^{2} \phi_{s}(\mathbf{r})\right] .
\end{aligned}
$$

Substituting Eqn. 32 and re-arranging terms, yields

$$
\left[\nabla^{2}+k_{0}^{2} n_{0}^{2}(\mathbf{r})\right]\left(\psi^{i n c}(\mathbf{r}) \phi_{s}(\mathbf{r})\right)=\psi^{i n c}(\mathbf{r})\left[2 \nabla \phi_{i}(\mathbf{r}) \cdot \nabla \phi_{s}(\mathbf{r})+\nabla^{2} \phi_{s}(\mathbf{r})\right] .
$$

Retaining only the first two terms in the expansion of Eqn. 31,

$$
\begin{aligned}
n^{2}(\mathbf{r}) & \approx n_{0}^{2}(\mathbf{r})+n_{1}(\mathbf{r}), \\
\phi(\mathbf{r}) & \approx \phi_{i}(\mathbf{r})+\phi_{1}^{s c a t}(\mathbf{r}) \equiv \phi_{i}(\mathbf{r})+\phi_{s}(\mathbf{r}),
\end{aligned}
$$

and using Eqn. 33, we obtain the distorted wave Rytov approximation,

$$
\left[\nabla^{2}+k_{0}^{2} n_{0}^{2}(\mathbf{r})\right]\left(\psi^{i n c}(\mathbf{r}) \phi_{s}(\mathbf{r})\right)=-k_{0}^{2} n_{1}(\mathbf{r}) \psi^{i n c}(\mathbf{r}) .
$$

Using Eqn. 20, we cast this into an integral equation

$$
\phi_{s}(\mathbf{r})=\frac{k_{0}^{2}}{\psi^{i n c}(\mathbf{r})} \int d \mathbf{r}^{\prime} G\left(\mathbf{r}, \mathbf{r}^{\prime}, \omega\right) n_{1}\left(\mathbf{r}^{\prime}\right) \psi^{i n c}\left(\mathbf{r}^{\prime}\right) .
$$

From Eqn. 39, we observe that

$$
n_{1}(\mathbf{r}) \approx n^{2}(\mathbf{r})-n_{0}^{2}(\mathbf{r})
$$

When $n_{0}(\mathbf{r}) \equiv 1$, Eqn. 41 reduces to the classical Rytov approximation,

$$
\phi_{s}(\mathbf{r})=\frac{k_{0}^{2}}{\psi^{\text {inc }}(\mathbf{r})} \int d \mathbf{r}^{\prime} G_{0}\left(\mathbf{r}, \mathbf{r}^{\prime}, \omega\right) o\left(\mathbf{r}^{\prime}\right) \psi^{\text {inc }}\left(\mathbf{r}^{\prime}\right) .
$$




\subsection{Summary of Born \& Rytov Approximations}

The forward scattering equations for the Born and Rytov approximations have identical forms for the quantity they approximate: the scattered amplitude for the former, and the scattered phase for the latter. We repeat them here,

$$
\begin{aligned}
\psi_{B}^{s c a t}\left(\mathbf{r}, \mathbf{R}_{n}^{t}, \omega\right) & =k_{0}^{2} \int d \mathbf{r}^{\prime} G_{0}\left(\mathbf{r}, \mathbf{r}^{\prime}, \omega\right) o\left(\mathbf{r}^{\prime}\right) \psi^{i n c}\left(\mathbf{r}^{\prime}, \mathbf{R}_{n}^{t}, \omega\right) \\
\phi_{s}(\mathbf{r}) & =\frac{k_{0}^{2}}{\psi^{\text {inc }}(\mathbf{r})} \int d \mathbf{r}^{\prime} G_{0}\left(\mathbf{r}, \mathbf{r}^{\prime}, \omega\right) o\left(\mathbf{r}^{\prime}\right) \psi^{i n c}\left(\mathbf{r}^{\prime}\right)
\end{aligned}
$$

We may compactly express these two equations as

$$
U^{s c a t}(\mathbf{r})=C(\mathbf{r}) k_{0}^{2} \int d \mathbf{r}^{\prime} G_{0}\left(\mathbf{r}, \mathbf{r}^{\prime}, \omega\right) o\left(\mathbf{r}^{\prime}\right) \psi^{i n c}\left(\mathbf{r}^{\prime}\right)
$$

where we have the following definitions

$$
\begin{aligned}
U^{\text {scatt }}(\mathbf{r}) & \equiv\left\{\begin{array}{cc}
\psi_{B}^{\text {scat }}\left(\mathbf{r}, \mathbf{R}_{n}^{t}, \omega\right) & \text { for the Born approximation, } \\
\phi_{s}(\mathbf{r}) & \text { for the Rytov approximation, }
\end{array}\right. \\
C(\mathbf{r}) & \equiv\left\{\begin{array}{cc}
1 & \text { for the Born approximation, } \\
\frac{1}{\psi^{\text {inc }}(\mathbf{r})} & \text { for the Rytov approximation. }
\end{array}\right.
\end{aligned}
$$

\subsection{Incident Fields}

Usually, the incident field is taken to be either a plane with unit propagation vector $\hat{\mathbf{s}}$ or spherical wave due to a point source located at $\mathbf{R}_{n}^{t}$. We express these mathematically as

$$
\psi^{i n c}(\mathbf{r})= \begin{cases}P(\omega) e^{i k_{0} \hat{\mathbf{s}} \cdot \mathbf{r}} & \text { plane wave, } \\ P(\omega) G_{0}\left(\mathbf{r}, \mathbf{R}_{n}^{t}, \omega\right) & \text { spherical wave }\end{cases}
$$

where $P(\omega)$ is the temporal spectrum of the incident field, and $G\left(\mathbf{r}, \mathbf{r}^{\prime}, \omega\right)$ is the Green function response of the medium.

We summarize the forward models of Eqn. 46 as follows:

$$
\begin{aligned}
U^{s c a t}(\mathbf{r}, \hat{\mathbf{s}}) & =C(\mathbf{r}) P(\omega) k_{0}^{2} \int d \mathbf{r}^{\prime} G_{0}\left(\mathbf{r}, \mathbf{r}^{\prime}, \omega\right) o\left(\mathbf{r}^{\prime}\right) e^{i k_{0} \hat{\mathbf{s}} \cdot \mathbf{r}^{\prime}}, \\
U^{s c a t}\left(\mathbf{r}, \mathbf{R}^{t}\right) & =C(\mathbf{r}) P(\omega) k_{0}^{2} \int d \mathbf{r}^{\prime} G_{0}\left(\mathbf{r}, \mathbf{r}^{\prime}, \omega\right) o\left(\mathbf{r}^{\prime}\right) G_{0}\left(\mathbf{r}^{\prime}, \mathbf{R}^{t}, \omega\right) .
\end{aligned}
$$

With our forward models in hand, we are now prepared to develop a method for inverting them to determine the scattering object based upon a set of scattered field measurements. We derive the "classical" plane wave diffraction tomography algorithm [9] in the following section. 


\section{Inversion Using Incident Plane Wave and Planar Measure- ment}

Eqns. 50 and 51 represent two possible forward models depending upon the nature of the probing source (either plane wave or point). The measurement system rotates about the fixed object. We label the fixed object coordinate system by $\mathbf{R} \equiv(X, Y, Z)$. The axis of rotation is the $Y$-axis. The rotated coordinate system is defined by $\mathbf{r} \equiv(x, y, z)=(-Z \sin \theta+X \cos \theta, 0, Z \cos \theta+X \sin \theta)$. The relationship between the two coordinate systems is given by

$$
\left[\begin{array}{l}
x \\
y \\
z
\end{array}\right]=\left[\begin{array}{ccc}
\cos \theta & 0 & -\sin \theta \\
0 & 1 & 0 \\
\sin \theta & 0 & \cos \theta
\end{array}\right]\left[\begin{array}{l}
X \\
Y \\
Z
\end{array}\right]
$$

We take the $z$-axis to be the major alignment axis of the measurement system. We assume a planar measurement surface located at $z=z_{p}$ whose normal lies along the $z$-axis:

$$
\mathbf{R}^{r}=\left(x, y, z_{p}\right)=\left(\mathbf{r}_{\perp}, z_{p}\right) .
$$

A schematic of the measurement system is presented in Figure 1.

For future reference, the complementary rotated spatial Fourier variables are defined by

$$
\begin{aligned}
\mathbf{k} \cdot \mathbf{r} & =k_{x} x+k_{y} y+k_{z} z, \\
& =k_{x}(X \cos \theta-Z \sin \theta)+k_{y} y+k_{z}(X \sin \theta+Z \cos \theta), \\
& =\left(k_{x} \cos \theta+k_{z} \sin \theta\right) X+k_{y} Y+\left(-k_{x} \sin \theta+k_{z} \cos \theta\right) Z, \\
& =\mathbf{K} \cdot \mathbf{R},
\end{aligned}
$$

so that we have

$$
\left[\begin{array}{l}
K_{x} \\
K_{y} \\
K_{z}
\end{array}\right]=\left[\begin{array}{ccc}
\cos \theta & 0 & \sin \theta \\
0 & 1 & 0 \\
-\sin \theta & 0 & \cos \theta
\end{array}\right]\left[\begin{array}{l}
k_{x} \\
k_{y} \\
k_{z}
\end{array}\right]
$$

\subsection{Plane Wave Details \& Fourier Diffraction Theorem}

The forward scattering model is given by the approximation of Eqn. 50:

$$
U^{s c a t}\left(\mathbf{R}^{r}, \hat{\mathbf{s}}\right)=C\left(\mathbf{R}^{r}\right) P(\omega) k_{0}^{2} \int d \mathbf{r}^{\prime} G_{0}\left(\mathbf{R}^{r}, \mathbf{r}^{\prime}, \omega\right) o\left(\mathbf{r}^{\prime}\right) e^{i k_{0} \hat{\mathbf{s}} \cdot \mathbf{r}^{\prime}},
$$

where we now explicitly state the background Green function

$$
G_{0}\left(\mathbf{R}^{r}, \mathbf{r}^{\prime}, \omega\right)=\frac{e^{i k_{0}\left|\mathbf{R}^{r}-\mathbf{r}^{\prime}\right|}}{4 \pi\left|\mathbf{R}^{r}-\mathbf{r}^{\prime}\right|}
$$




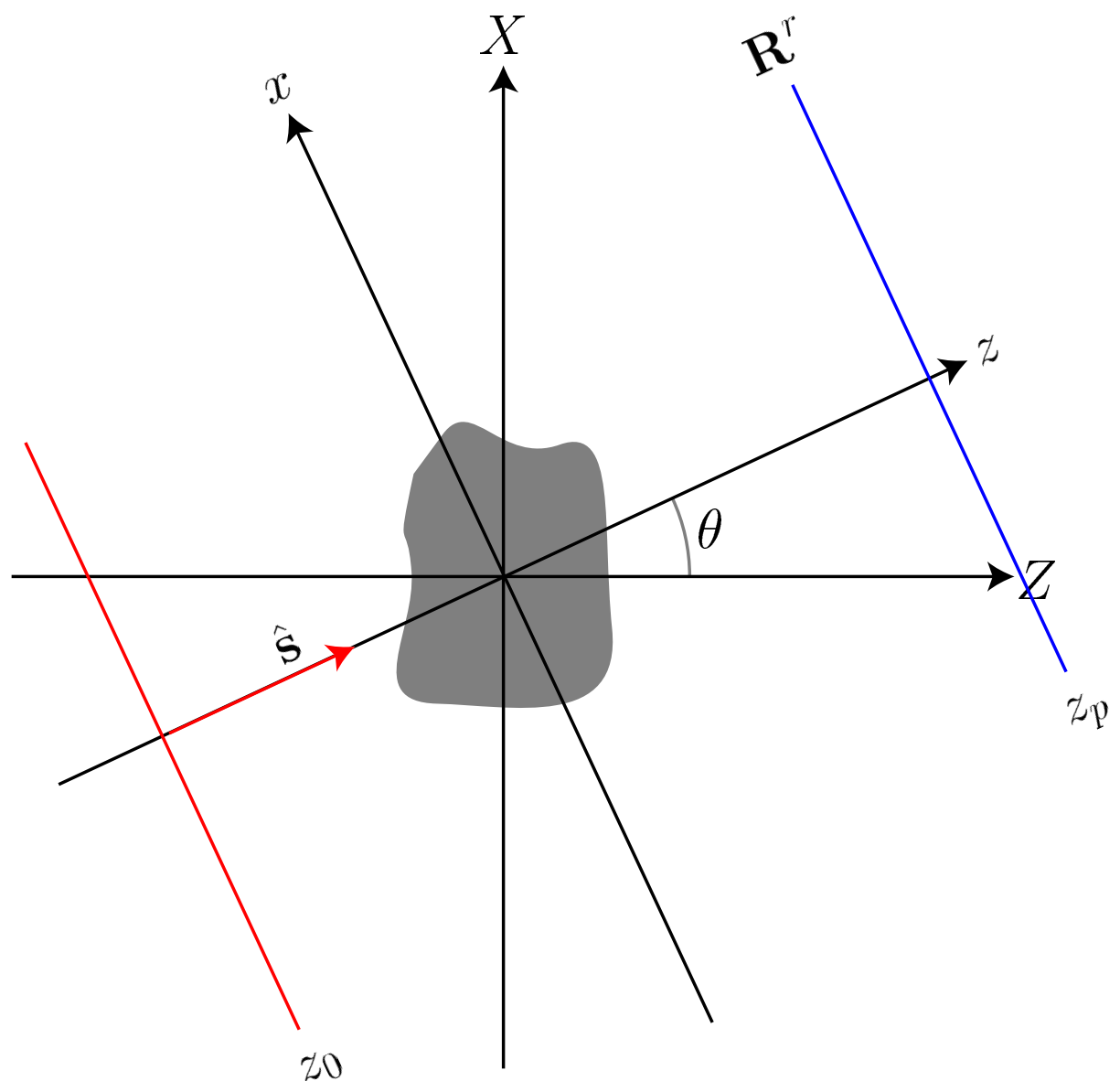

$$
\begin{gathered}
\mathbf{R}^{r}=\underbrace{\left(x, y, z_{p}\right)=\left(\mathbf{r}_{\perp}, z_{p}\right)}_{\text {rotated }} \\
\hat{\mathbf{s}}=\underbrace{(0,0,1)}_{\text {rotated }}=\underbrace{(\sin \theta, 0, \cos \theta)}_{\text {fixed }} \\
\mathbf{R}^{t}=\underbrace{\left(0,0, z_{0}\right)}_{\text {rotated }}=\underbrace{z_{0}(\sin \theta, 0, \cos \theta)}_{\text {fixed }}
\end{gathered}
$$

Figure 1: Schematic of measurement system. The plane wave propagation vector, $\hat{\mathrm{s}}$, is given by $(\sin \theta, 0, \cos \theta)$ in the fixed object coordinate system. The point source location is given by $z_{0}(\sin \theta, 0, \cos \theta)$ in the fixed coordinate system. The entire measurement system rotates about the $y$-axis which is out of the plane of the page. 
and

$$
k_{0} \equiv \frac{\omega}{c_{0}}
$$

is the background wave number. Substitute the Weyl expansion,

$$
\frac{e^{i k_{0}\left|\mathbf{R}^{r}-\mathbf{r}^{\prime}\right|}}{4 \pi\left|\mathbf{R}^{r}-\mathbf{r}^{\prime}\right|}=\frac{i}{8 \pi^{2}} \int d \mathbf{k}_{\perp}^{\prime} \frac{e^{i\left(\mathbf{k}_{\perp}^{\prime} \cdot\left(\mathbf{r}_{\perp}-\mathbf{r}_{\perp}^{\prime}\right)+\gamma(\omega)\left|z_{p}-z^{\prime}\right|\right)}}{\gamma(\omega)}
$$

where $\gamma(\omega) \equiv \sqrt{k_{0}^{2}-\left|\mathbf{k}_{\perp}^{\prime}\right|^{2}}$, and $\mathbf{R}^{r} \equiv\left(\mathbf{r}_{\perp}, z_{p}\right)$, into Eqn. 55

$$
U^{s c a t}\left(\mathbf{R}^{r}, \hat{\mathbf{s}}\right)=\frac{i C\left(\mathbf{R}^{r}\right) P(\omega) k_{0}^{2}}{8 \pi^{2}} \int d \mathbf{r}^{\prime} \int d \mathbf{k}_{\perp}^{\prime} \frac{e^{i \mathbf{k}_{\perp}^{\prime} \cdot\left(\mathbf{r}_{\perp}-\mathbf{r}_{\perp}^{\prime}\right)+\gamma(\omega)\left|z_{p}-z^{\prime}\right|}}{\gamma(\omega)} o\left(\mathbf{r}^{\prime}\right) e^{i k_{0}(\omega) \hat{\mathbf{s}} \cdot \mathbf{r}^{\prime}} .
$$

We note that for the transmission mode measurement system depicted in Figure $1, z_{p}>z^{\prime}$ so that we may set

$$
\left|z_{p}-z^{\prime}\right|=z_{p}-z^{\prime}
$$

to achieve

$$
\begin{aligned}
U^{\text {scat }}\left(\mathbf{R}^{r}, \hat{\mathbf{s}}\right)= & \frac{i C\left(\mathbf{R}^{r}\right) P(\omega) k_{0}^{2}}{8 \pi^{2}} \int d \mathbf{r}^{\prime} \int d \mathbf{k}_{\perp}^{\prime} \frac{e^{i\left(\mathbf{k}_{\perp}^{\prime} \cdot\left(\mathbf{r}_{\perp}-\mathbf{r}_{\perp}^{\prime}\right)+\gamma(\omega)\left(z_{p}-z^{\prime}\right)\right)}}{\gamma(\omega)} o\left(\mathbf{r}^{\prime}\right) e^{i k_{0}(\omega) z^{\prime}}, \\
= & \frac{i C\left(\mathbf{R}^{r}\right) P(\omega) k_{0}^{2}}{8 \pi^{2}} \int \frac{d \mathbf{k}_{\perp}^{\prime}}{\gamma(\omega)} e^{i\left(\mathbf{k}_{\perp}^{\prime} \cdot \mathbf{r}_{\perp}+\gamma(\omega) z_{p}\right)} \times \\
& \int d \mathbf{r}^{\prime} o\left(\mathbf{r}^{\prime}\right) e^{-i\left(\mathbf{k}_{\perp}^{\prime} \cdot \mathbf{r}_{\perp}^{\prime}-\left(k_{0}(\omega)-\gamma(\omega)\right) z^{\prime}\right)} .
\end{aligned}
$$

Using the spatial Fourier transform pair definitions from Appendix A,

$$
\begin{aligned}
\tilde{\psi}(\mathbf{k}) & =\int d \mathbf{r} \psi(\mathbf{r}) e^{-i \mathbf{k} \cdot \mathbf{r}} \\
\psi(\mathbf{r}) & =\frac{1}{(2 \pi)^{n}} \int d \mathbf{k} \tilde{\psi}(\mathbf{k}) e^{i \mathbf{k} \cdot \mathbf{r}}
\end{aligned}
$$

where $n$ is the dimensionality of the transform, we conclude the $\mathbf{r}^{\prime}$ integral in Eqn. 61 is the spatial Fourier transform of the object:

$$
O\left(\mathbf{k}_{\perp}^{\prime}, k_{z}^{\prime}\right)=\int d \mathbf{r}^{\prime} o\left(\mathbf{r}^{\prime}\right) e^{-i\left(\mathbf{k}_{\perp}^{\prime} \cdot \mathbf{r}_{\perp}^{\prime}-\left(k_{0}-\gamma(\omega)\right) z^{\prime}\right)},
$$

where $k_{z}^{\prime} \equiv-\left(k_{0}-\gamma(\omega)\right)$. Eqn. 61 then reads

$$
U^{s c a t}\left(\mathbf{r}_{\perp}, z_{p}, \hat{\mathbf{s}}\right)=\frac{i C\left(\mathbf{R}^{r}\right) P(\omega) k_{0}^{2}}{8 \pi^{2}} \int \frac{d \mathbf{k}_{\perp}^{\prime}}{\gamma(\omega)} e^{i\left(\mathbf{k}_{\perp}^{\prime} \cdot \mathbf{r}_{\perp}+\gamma(\omega) z_{p}\right)} O\left(\mathbf{k}_{\perp}^{\prime}, k_{z}^{\prime}\right) .
$$


We digress briefly to examine the $C\left(\mathbf{R}^{r}\right)$ factor under the Born and Rytov approximations. We have

$$
C\left(\mathbf{R}^{r}\right)=\left\{\begin{array}{cl}
1 & \text { for the Born approximation, } \\
\frac{1}{\psi^{i n c}(\mathbf{r})}=\frac{e^{-i k_{0} \hat{\mathbf{s}} \cdot \mathbf{R}^{r}}}{P(\omega)}=\frac{e^{-i k_{0} z_{p}}}{P(\omega)} & \text { for the Rytov approximation. }
\end{array}\right.
$$

We note $C\left(\mathbf{R}^{r}\right)$ is independent of the planar measurement variable $\mathbf{r}_{\perp}$. This is significant because we now perform a planar Fourier transform (PFT) of the measured scattered quantity ${ }^{1}$,

$$
\begin{aligned}
\bar{U}^{\text {scat }}\left(\mathbf{k}_{\perp}, z_{p}, \hat{\mathbf{s}}\right)= & \int d \mathbf{r}_{\perp} U^{\text {scat }}\left(\mathbf{r}_{\perp}, z_{p}, \hat{\mathbf{s}}\right) e^{-i \mathbf{k}_{\perp} \cdot \mathbf{r}_{\perp}}, \\
= & \frac{i C\left(\mathbf{R}^{r}\right) P(\omega) k_{0}^{2}}{8 \pi^{2}} \times \\
& \int \frac{d \mathbf{k}_{\perp}^{\prime}}{\gamma(\omega)}\left[\int d \mathbf{r}_{\perp} e^{i\left(\mathbf{k}_{\perp}^{\prime}-\mathbf{k}_{\perp}\right) \cdot \mathbf{r}_{\perp}}\right] e^{i \gamma(\omega) z_{p}} O\left(\mathbf{k}_{\perp}^{\prime}, k_{z}^{\prime}\right) .
\end{aligned}
$$

We use the identity

$$
\delta\left(\mathbf{k}_{\perp}^{\prime}-\mathbf{k}_{\perp}\right)=\frac{1}{(2 \pi)^{n-1}} \int d \mathbf{r}_{\perp} e^{i\left(\mathbf{k}_{\perp}^{\prime}-\mathbf{k}_{\perp}\right) \cdot \mathbf{r}_{\perp}}
$$

to reduce Eqn. 67 to

$$
\bar{U}^{\text {scat }}\left(\mathbf{k}_{\perp}, z_{p}, \hat{\mathbf{s}}\right)=\frac{i C\left(\mathbf{R}^{r}\right) P(\omega) k_{0}^{2} e^{i \gamma(\omega) z_{p}}}{2 \gamma(\omega)} O\left(\mathbf{k}_{\perp}, k_{z}\right) .
$$

Eqn. 69 relates the $n$-dimensional Fourier transform of the object to the $(n-1)$-dimensional planar transform of the measured scattered field (Born approximation) or the measured scattered phase (Rytov approximation). It is known as the Fourier Diffraction Theorem in wave-based tomography and is the equivalent of the Fourier Slice Theorem [10] of straight-ray tomography. Explicitly, we have the measured field (phase) on the $\mathbf{k}_{\perp}$ plane which is proportional to the object Fourier transform in the $\left(\mathbf{k}_{\perp}, k_{z}\right)$ space. This appears at first to be a mapping from an $n$-dimensional function to a $(n-1)$-dimensional function. Note, however, that $k_{z}$ depends upon $\mathbf{k}_{\perp}$ via

$$
k_{z} \equiv-\left(k_{0}-\sqrt{k_{0}^{2}-\left|\mathbf{k}_{\perp}\right|^{2}}\right)
$$

Thus, the relationship is a mapping of the $(n-1)$-dimensional locus of points through the $n$ dimensional space described by $\left(\mathbf{k}_{\perp}, k_{z}\right)$, to the $(n-1)$-dimensional Fourier space measurement plane. Applying the coordinate rotation of Eqn. 54 to account for all measurement angles $\theta$, we

\footnotetext{
${ }^{1}$ Amplitude in the Born approximation or phase in the Rytov approximation
} 
have in the fixed object frame

$$
\begin{aligned}
{\left[\begin{array}{l}
K_{x} \\
K_{y} \\
K_{z}
\end{array}\right] } & =\left[\begin{array}{ccc}
\cos \theta & 0 & \sin \theta \\
0 & 1 & 0 \\
-\sin \theta & 0 & \cos \theta
\end{array}\right]\left[\begin{array}{c}
k_{x} \\
k_{y} \\
-\left(k_{0}-\gamma(\omega)\right)
\end{array}\right] \\
= & {\left[\begin{array}{c}
k_{x} \cos \theta+\left(k_{0}-\gamma(\omega)\right) \sin \theta \\
k_{y} \\
-k_{x} \sin \theta-\left(k_{0}-\gamma(\omega)\right) \cos \theta
\end{array}\right], }
\end{aligned}
$$

where we have explicitly used the dependence of $k_{z}$ on $k_{0}$ and $\mathbf{k}_{\perp} \equiv\left(k_{x}, k_{y}\right)$. Curves of $K_{x}\left(k_{x}\right)$ and $K_{z}\left(k_{x}\right)$ are presented in Figure 2 for multiple values of $\theta$ with $\left|k_{x}\right| \leq k_{0}$. This constraint on $k_{x}$, or more generally the constraint that $\left|\mathbf{k}_{\perp}\right| \leq k_{0}$ is required to ensure $k_{z}$ remain real valued as can be observed in Eqn. 70. For $\left|\mathbf{k}_{\perp}\right|>k_{0}, k_{z}$ becomes complex and the resulting fields evanescent. Although evanescent field tomography is possible, given the dimensions of the measurement system with respect to the illuminating wavelength, it is not practical because no evanescent field will reach the measurement plane.

Although our entire development has been in $n$-dimensions (for all intents, three dimensions), the Fourier Diffraction Theorem is entirely two dimensional. Eqn. 71 shows the relationship between the $K_{y}$ and $k_{y}$ dimensions to be one-to-one. That is, the mapping is one-to-one in the Fourier variable complementary to the axis of rotation. Thus, in the three Fourier dimensions, the loci of points plotted in Figure 2 form a cylinder along the $k_{y}$ axis, perpendicular to the plane of the page.

The Fourier Diffraction Theorem dictates the resolution of the reconstruction. Assume evanescent fields are neglected, the resolution limit is governed by the Ewald sphere $[8,9]$ which indicates the transition from propagating to evanescent fields. For this case of narrow-band transmission mode tomography, this limit is inversely proportional to $\sqrt{2} k_{0}$. Consider the spatial frequency limit in inverse length (as opposed to radians),

$$
\begin{aligned}
\sqrt{2} \eta & \equiv \sqrt{2} \sqrt{\eta_{x}^{2}+\eta_{z}^{2}} \\
& =\sqrt{2} \frac{k_{0}}{2 \pi} \\
& =\sqrt{2} \frac{f}{c_{0}} \\
& =\frac{\sqrt{2}}{\lambda}
\end{aligned}
$$

where we have used Eqn. 57, $\omega=2 \pi f$, and $c_{0}=f \lambda$, where $f$ is the frequency of the illuminating source. The spatial resolution limit is thus $\lambda / \sqrt{2}$ in the plane of rotation. In the perpendicular domain, the resolution limit is governed by the spatial sampling along the $y$-axis.

We are now prepared to invert Eqn. 69 in order to "reconstruct" the scattering object. In theory it is a simple matter of solving for the object spectrum and performing an inverse Fourier transform. However, we do not know the object's spectrum on a Cartesian grid: we know it at the points defined by Eqn. 71. Furthermore, lower spatial frequencies are sampled more densely by the Fourier Diffraction Theorem than higher ones, with the zero frequency (that is, DC) being sampled 


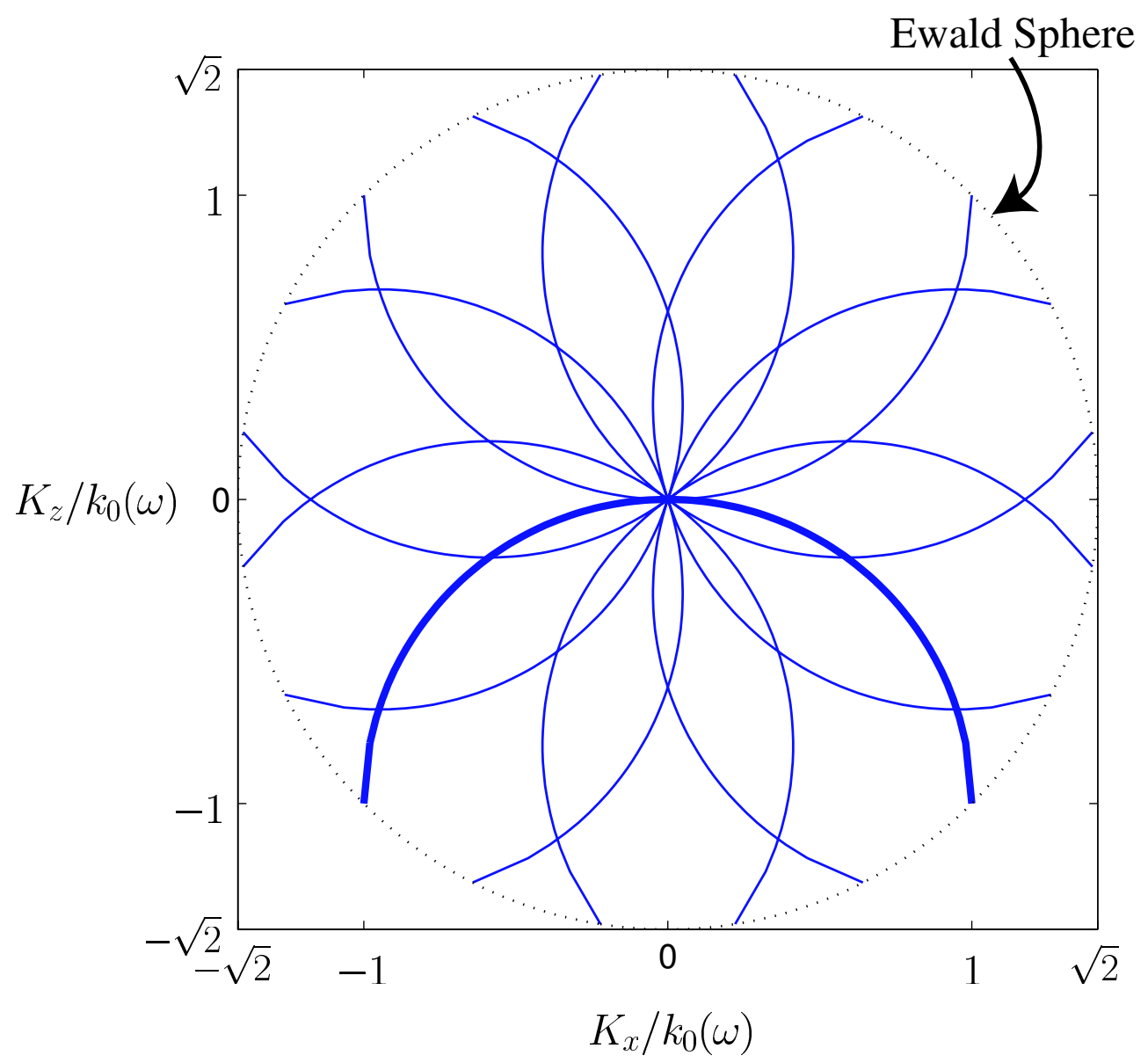

Figure 2: Graphical representation of the Fourier Diffraction Theorem of Eqn. 69. The curves represent the loci of points given by Eqn. 71 in the object's Fourier space. The Ewald sphere defines the transition from propagating to evanescent field spectra.

the most: all curves pass through the origin. We account for these issues and develop an inverse in the next section.

\subsection{Plane Wave Inversion}

Solving for the object spectrum of Eqn. 69 yields

$$
O\left(\mathbf{k}_{\perp}, k_{z}\right)=-\frac{i 2 \gamma(\omega) e^{-i \gamma(\omega) z_{p}}}{C\left(\mathbf{R}^{r}\right) P(\omega) k_{0}^{2}} \bar{U}^{s c a t}\left(\mathbf{k}_{\perp}, z_{p}, \hat{\mathbf{s}}\right)
$$

in terms of the PFT of the measured scattered field.

For practical considerations, we neglect the evanescent field information and limit ourselves to the propagating spectrum of $\left|\mathbf{k}_{\perp}\right| \leq k_{0}$. This yields a low pass reconstruction of the object as 
indicated by the spectral coverage of Figure 2. We define the unit step function by

$$
S(k) \equiv \begin{cases}0 & k<0 \\ 1 & k \geq 0\end{cases}
$$

and write

$$
O_{l p}\left(\mathbf{k}_{\perp}, k_{z}\right)=-\frac{i 2 \gamma(\omega) e^{-i \gamma(\omega) z_{p}}}{C\left(\mathbf{R}^{r}\right) P(\omega) k_{0}^{2}} \bar{U}^{s c a t}\left(\mathbf{k}_{\perp}, z_{p}, \hat{\mathbf{s}}\right) S\left(k_{0}-\left|\mathbf{k}_{\perp}\right|\right),
$$

where the $l p$ subscript indicates we will be performing a low pass reconstruction.

We are now prepared to define the inverse Fourier transform of Eqn. 75 to obtain the low pass object reconstruction. The inverse Fourier transform is

$$
\hat{o}(\mathbf{R})=\frac{1}{(2 \pi)^{n}} \int d \mathbf{K} O_{l p}(\mathbf{K}) e^{i \mathbf{K} \cdot \mathbf{R}},
$$

where $\mathbf{K}$ is given by Eqn. 71. The object spectrum of Eqn. 76 is specified in terms of the Cartesian coordinate system given by $\mathbf{K}=\left(K_{x}, K_{y}, K_{z}\right)$. The actual measured values of the spectrum are known along the arcs given by

$$
\left[\begin{array}{l}
K_{x} \\
K_{y} \\
K_{z}
\end{array}\right]=\left[\begin{array}{c}
k_{x} \cos \theta+\left(k_{0}-\gamma(\omega)\right) \sin \theta \\
k_{y} \\
-k_{x} \sin \theta-\left(k_{0}-\gamma(\omega)\right) \cos \theta
\end{array}\right]
$$

In order to perform the integral of Eqn. 76, we must change the integration variables from the object spectrum's Cartesian frame to those of the rotated frame of the incident plane wave. The Jacobian of the transformation is

$$
J(\mathbf{K} ; \mathbf{k})=\left|\begin{array}{lll}
\partial K_{x} / \partial k_{x} & \partial K_{y} / \partial k_{x} & \partial K_{z} / \partial k_{x} \\
\partial K_{x} / \partial k_{y} & \partial K_{y} / \partial k_{y} & \partial K_{z} / \partial k_{y} \\
\partial K_{x} / \partial k_{z} & \partial K_{y} / \partial k_{z} & \partial K_{z} / \partial k_{z}
\end{array}\right|=\frac{k_{0} k_{x}}{\gamma(\omega)} .
$$

We now express the inverse Fourier transform as

$$
\hat{o}(\mathbf{R})=\frac{-i 2}{(2 \pi)^{n} C\left(\mathbf{R}^{r}\right) P(\omega) k_{0}} \int_{\left|\mathbf{k}_{\perp}\right|<k_{0}} d \mathbf{k}_{\perp}\left|k_{x}\right| e^{-i \gamma(\omega) z_{p}} \int_{0}^{2 \pi} d \theta \bar{U}^{s c a t}\left(\mathbf{k}_{\perp}, z_{p}, \hat{\mathbf{s}}\right) e^{i \mathbf{k} \cdot \mathbf{r}},
$$

where we have used Eqns. 54 and 73.

$$
\hat{o}(\mathbf{R})=\frac{-i 2 e^{-i k_{0} z_{p}}}{(2 \pi)^{n} C\left(\mathbf{R}^{r}\right) P(\omega) k_{0}} \int_{0}^{2 \pi} d \theta \int_{-\infty}^{\infty} d \mathbf{k}_{\perp} H\left(\mathbf{k}_{\perp}, z-z_{p}\right) \bar{U}^{s c a t}\left(\mathbf{k}_{\perp}, z_{p}, \hat{\mathbf{s}}\right) e^{i \mathbf{k}_{\perp} \cdot \mathbf{r}_{\perp}}
$$

where

$$
H\left(\mathbf{k}_{\perp}, z\right) \equiv\left|k_{x}\right| e^{-i\left(k_{0}-\gamma(\omega)\right) z} S\left(k_{0}-\left|\mathbf{k}_{\perp}\right|\right)
$$


Eqn. 80 is the "standard" filtered backpropagation algorithm [9]. The $\left|k_{x}\right|$ expression represents a filter which accounts for the non-uniform sampling of the Fourier Diffraction Theorem. The product $e^{-i k_{0} z_{p}} e^{-i\left(k_{0}-\gamma(\omega)\right) z}$ represents a plane-to-plane backpropagation of the measured quantity from the measurement plane to a plane within the object. Finally, the $S\left(k_{0}-\left|\mathbf{k}_{\perp}\right|\right)$ expression imposes the low-pass limit of the reconstruction require to remove evanescent fields which, when backpropagated, diverge given their exponentially decaying nature in the forward propagation direction.

\section{Summary \& Conclusions}

We have developed a wave-based inversion and imaging algorithm which is applicable to the Optical Quadrature Microscope. We have assumed narrow-band plane wave illumination of the object under evaluation. We have also assumed multiple planar measurements, uniformly distributed $360^{\circ}$ about the object, are possible while maintaining a fixed object to measurement plane distance. 


\section{References}

[1] D. O. Hogenboom and C. A. DiMarzio. Quadrature detection of a doppler signal. Applied Optics, 13(13):2569-2572, May 1998.

[2] D. O. Hogenboom, C. A. DiMarzio, T. J. Gaudette, A. J. Devaney, and S. C. Lindberg. Threedimensional images generated by quadrature interferometry. Optical Society of America, 23(10):783-785, May 1998.

[3] Y. Glina, G. A. Tsihrintzis, C. M. Warner, D. O. Hogenboom, and C. A. DiMarzio. On the use of the optical quadrature method in tomographic microscopy. In SPIE Conference on Three-Dimensional and Multidimensional Microscopy: Image Acquisition and Processing VI, volume 3605, pages 101-106. SPIE, January 1999.

[4] D. J. Townsend, K. D. Quarles, A. L. Thomas, W. S. Rockward, C. M. Warner, J. A. Newmark, and C. A. DiMarzio. Quantitative phase measurements using a quadrature tomographic microscope. In T. Wilson J.-A. Conchello, C. J. Cogswell, editor, Three-Dimensional and Multidimensional Microscopy: Image Acquisition and Processing X, volume 4964, pages 59-65. SPIE, 2003.

[5] II W. C. Warger. The fusion of optical quadrature and differential interference contrast microscopy to produce accurate cell counts in live mouse embryos. Master's thesis, Northeastern University, 2005.

[6] J. D. Jackson. Classical Electrodynamics. John Wiley \& Sons, Inc., 3nd edition, 1999. ISBN 0-471-30932-X.

[7] G. Beylkin and M. L. Oristaglio. Distorted-wave born and distorted-wave rytov approximations. Optics Communications, 53(4):213-216, March 1985.

[8] M. Born and E. Wolf. Principles of Optics. Cambridge University Press, 7th edition, 1999. ISBN 0-521-642221.

[9] A. J. Devaney. A filtered backpropagation algorithm for diffraction tomography. Ultrasonic Imaging, 4(4):336-350, October 1982.

[10] A. C. Kak and M. Slaney. Principles of Computerized Tomographic Imaging. IEEE Press, 1988. ISBN 0-7803-0447-0. 


\section{A Standard Fourier Transforms}

We summarize without comment our Fourier transform definitions.

\section{Forward in time}

$$
U(\mathbf{r}, \omega)=\int_{-\infty}^{\infty} d t u(\mathbf{r}, t) e^{i \omega t}
$$

Inverse in time

$$
u(\mathbf{r}, t)=\frac{1}{2 \pi} \int_{-\infty}^{\infty} d \omega U(\mathbf{r}, \omega) e^{-i \omega t}
$$

Forward in space

$$
\tilde{U}(\mathbf{k}, \omega)=\int_{-\infty}^{\infty} d \mathbf{r} U(\mathbf{r}, \omega) e^{-i \mathbf{k} \cdot \mathbf{r}}
$$

Inverse in space

$$
U(\mathbf{r}, \omega)=\frac{1}{(2 \pi)^{3}} \int_{-\infty}^{\infty} d \mathbf{k} \tilde{U}(\mathbf{k}, \omega) e^{i \mathbf{k} \cdot \mathbf{r}}
$$

